\title{
Impulse-response functions of several detectors used in flow-injection analysis
}

\author{
I.C. van Nugteren-Osinga, E. Hoogendam, M. Bos and W.E. van der Linden * \\ University of Twente, Laboratory for Chemical Analysis-CT, P.O. Box 217, NL-7500 AE Enschede (The Netherlands)
}

(Received 24th April 1990)

\begin{abstract}
A procedure for the determination of the impulse-response function of a detector is given. Its application to photometers, ion-sensitive field effect transistors, a potentiometric detector at constant current and a voltammetric detector shows that the impulse-response function can be used to obtain specific information about the performance of the detector in the manifold. This function clearly shows the contribution of the detector to the peak broadening and how the detector generates the final signal from the presented concentration profile. From this information one could derive improvements to the detector, such as changing the construction of the detector cell, minimizing the influence of other parts of the manifold or adapting the attached electronics.
\end{abstract}

Keywords: Detectors; Ion-sensitive field effect transistors; Impulse-response functions

Several kinds of detectors can be and are used in flow-injection analysis. A detector is suitable for this purpose when it meets the requirements that it must have a small effective volume and a fast response which is linear over a large concentration range, and the attached electronics should not adversely affect the quality of the signal. Two related features of primary importance concerning detectors are their contributions to the peak broadening and to the detection limits.

Specific information on a detector can be obtained by determining its impulse-response function. This function represents the contribution of the detector to the peak broadening and may give an indication of how the detector generates the final signal from the presented concentration profile. From this information one can conclude how the construction of the detector cell can be improved. With the determination of the detector impulse-response function, coloured noise, which may be caused, for example, by the pump or the attached electronics, can be tracked down. By eliminating the sources of coloured noisc and changing the construction of the detector cell, the signal-to-noise ratio can be improved.

Finally, it is possible to use the impulse-response function of the detector, as one of the elements, for calculating the final signal of a complete manifold. This can be done by convoluting the impulse-response functions of the separate modules [1].

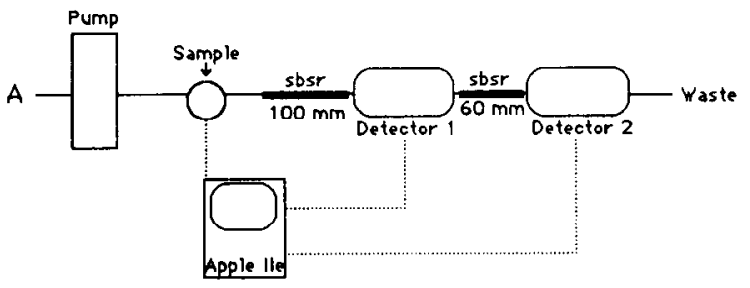

Fig. 1. The manifold used. Sulid lines, fluid streams; dotted lines, electrical connections. Sbsr = single-bead string reactor. 


\section{EXPERIMENTAL}

\section{Method}

The manifold shown in Fig. 1 is used for the determination of the impulse-response function of a detector. By deconvoluting the signal of detector $2[g(t)]$ by the signal of reference detector 1 [ $f(t)]$, the impulse-response function of detector 2 is found. This procedure can be depicted mathematically as

$$
\begin{aligned}
f(t) \stackrel{\mathrm{FFT}}{\longrightarrow} F(f) & \\
g(t) \stackrel{\mathrm{FFT}}{\longrightarrow} G(f) & \rightarrow \frac{G(f) F(f)^{*}}{F(f) F(f)^{*}+k} \\
& =H(f) \stackrel{\text { inv.FFT }}{\rightarrow} h(t)
\end{aligned}
$$

where FFT means fast Fourier transform, $F(f)^{*}$ is the complex conjugate of $F(f), k$ is a constant which is related to the noise [this value is responsible for the filtering (Hunt filter)], $H(f)$ is the transform function, inv.FFT is the inverse fast Fourier transform and $h(t)$ is the impulse-response function $[1,2]$.

A conductivity cell is chosen as reference detector 1 . This cell has a small effective volume and the attached electronics allow a fast response. The platinum electrodes are located on opposite sides and directly facing each other in the flow-through cell.

According to a study by Taylor and Nieman [3] on the effect of conductivity cell geometrics, it is possible to reach a good signal-to-noise ratio with this configuration. It is assumed that a manifold can be described by a tanks-in-series model [4] leading to a skewed peak-like signal. Because of its favourable performance it can be expected that the conductivity cell correctly reflects the presented concentration profile leading to a signal which matches this tanks-in-series model. Detector 2 and the connection between the detectors change the concentration profile showed by detector 1 . The impulse-response function reflects this alteration. The influence of the connection between the detector in the impulse-response function is small. The detectors of which the impulseresponse functions are determined are listed in Table 1. It is possible to compare the impulse-response functions of the different detectors, because in each instance the same connection is used.

The quality of the impulse-response function depends greatly on the number of points used for its calculation; for practical reasons, related to the capacity of the available memory of the computer, this number is limited in this case to 512 points.

\section{TABLE 1}

Detectors used

Spectrophotometer

Spectrophotometer

$\mathrm{pH}$ electrode

Wall-jet configuration used in potentiometric mode at constant current

Potassium ISFET [6]

Sodium ISFET

Used as reference detector 1 :

Conductivity cell
Skalar $6000,520-\mathrm{nm}$ filter

Zeiss PMQ III, variable wavelength with Hellma 80- $\mu$ l flow cell

Metrohm flow cell with a Radiometer (G2222c), glass electrode; reference electrode, calomel; attached electronics, Knick Model 763 microprocessor pH meter.

Metrohm flow cell with glassy carbon electrode and a gold electrode; attached electronics, in-house design

Flow cell [7]; attached electronics, ISFET type 2 amplifier, Electro-Medical Instrumentation. The drain current was fixed at $100 \mu \mathrm{A}$; sensitivity, 57 $\mathrm{mV}$ per decade

Flow cell [7]; attached electronics, ISFET type 2 amplifier, Electro-Medical Instrumentation. The drain current was fixed at $100 \mu \mathrm{A}$; sensitivity, 55 $\mathrm{mV}$ per decade.

Cell dimensions, length $25 \mathrm{~mm} \times 1.2 \mathrm{~mm}$ i.d.; effective volume, $2.5 \mu \mathrm{l}$; platinum electrodes in opposing configuration directly facing each other; attached electronics, Philips PW 9509 
TABLE 2

Carrier and sample solutions used

\begin{tabular}{|c|c|c|c|c|c|c|}
\hline \multirow[t]{2}{*}{ Solution } & \multicolumn{6}{|l|}{ Detector } \\
\hline & $\begin{array}{l}\text { Skalar } \\
\text { spectro- } \\
\text { photometer }\end{array}$ & $\begin{array}{l}\text { Zeiss } \\
\text { spectro- } \\
\text { photometer }\end{array}$ & $\begin{array}{l}\text { Wall-jet } \\
\text { system }\end{array}$ & $\begin{array}{l}\text { Glass pH } \\
\text { electrode }\end{array}$ & $\begin{array}{l}\text { Potassium } \\
\text { ISFET }\end{array}$ & $\begin{array}{l}\text { Sodium } \\
\text { ISFET }\end{array}$ \\
\hline Carrier & Water & Water & Water & Water & $\mathrm{KCl}^{\mathrm{a}}$ & $\mathrm{NaCl}^{\mathrm{a}}$ \\
\hline Analyte & $\mathrm{KMnO}_{4}$ & $\mathrm{KMnO}_{4}$ & $\mathrm{FeCl}_{3}$ & $\mathrm{HCl}$ & $\mathrm{KCl}$ & $\mathrm{NaCl}$ \\
\hline \multirow[t]{4}{*}{ Concentration of analyte $\left(\times 10^{-4} \mathrm{M}\right)$} & 1.8 & 0.5 & 2.50 & 5.00 & 100.0 & 100.0 \\
\hline & 1.2 & 1.0 & 1.25 & 3.75 & 75.0 & 75.0 \\
\hline & 0.9 & 1.5 & 0.62 & 2.50 & 50.0 & 50.0 \\
\hline & 0.6 & 2.0 & & 1.25 & 25.0 & 25.0 \\
\hline
\end{tabular}

$\overline{1 \times 10^{-3}} \mathrm{M}$.

The sample rate is chosen in such a way that parts of the detector signal with the highest rate of change are adequately represented after the analogue to digital conversion (error $<0.5$ least significant bit).

By representing the transformed signal of the detector in a magnitude spectrum, coloured noise can be tracked down. In a magnitude spectrum, the quantity $\sqrt{I r^{2}+R e^{2}}$, in where $I r$ and $R e$ are the imaginary and real part of the signal in the Fourier domain, respectively, is plotted against the frequency. The noise contribution is mainly found in the high-frequency part of this spectrum. Coloured noise is visible as distinct peaks. By eliminating the origin of coloured noise it is possible to improve the signal-to-noise ratio.

In the determination of the impulse-response function, it is also important to eliminate the white noise as far as possible. This is done by means of the Hunt filter [2].

\section{Chemicals}

All chemicals were of analytical-reagent grade. The carrier and sample solutions are given in Table 2.

\section{Apparatus}

The manifold used is depicted schematically in Fig. 1. It consists of a peristaltic pump (Gilson Minipuls II), an injection valve of a rotary type (Rheodyne) and PTFE tubing. The injection volume was about $100 \mu \mathrm{l}$. Two single-bead string reactors $(100 \mathrm{~mm}$ and $60 \mathrm{~mm} \times 1.2 \mathrm{~mm}$ i.d., bead diameter $0.65-0.90 \mathrm{~mm}$ ) were used to accomplish enhanced radial mixing wherever required, i.e., just before the detectors. The injection valve was controlled and the data were collected by the computer. Specifications of the detectors are listed in Table 1. The curves were measured with different sample concentrations (Table 2), different

\section{TABLE 3}

Flow-rates $\left(\mathrm{ml} \mathrm{min}^{-1}\right)$ used for the determination of the impulse-response functions of the detectors

\begin{tabular}{llllll} 
Detector & & & & \\
\hline $\begin{array}{l}\text { Skalar } \\
\text { spectrophotometer }\end{array}$ & $\begin{array}{l}\text { Zeiss } \\
\text { spectrophotometer }\end{array}$ & $\begin{array}{l}\text { Wall-jet } \\
\text { systcm }\end{array}$ & $\begin{array}{l}\text { Glass pH } \\
\text { clcctrode }\end{array}$ & $\begin{array}{l}\text { Potassium } \\
\text { ISFET }\end{array}$ & $\begin{array}{l}\text { Sodium } \\
\text { ISFET }\end{array}$ \\
\hline 0.40 & 0.79 & 0.30 & 0.20 & 0.92 & 0.92 \\
0.53 & 0.92 & 0.65 & 0.38 & 1.05 & 1.05 \\
0.66 & 1.05 & 1.00 & 0.59 & 1.18 & 1.18 \\
0.79 & 1.18 & 1.36 & 1.00 & 2.40 & 2.70
\end{tabular}


flow-rates (Table 3) and with different sample rates. The units on the ordinate are a measure for the voltage derived from the detector; $1 \mathrm{E}$ is 0.48
$\mathrm{mV}$. An Apple IIe computer with an $A / D$ converter, Digiloc, 12 bits $( \pm 1 \mathrm{~V})$, and a $\mathrm{D} / \mathrm{A}$ converter, Kronemuis, 12 bits $(0-10 \mathrm{~V})$, was used. In
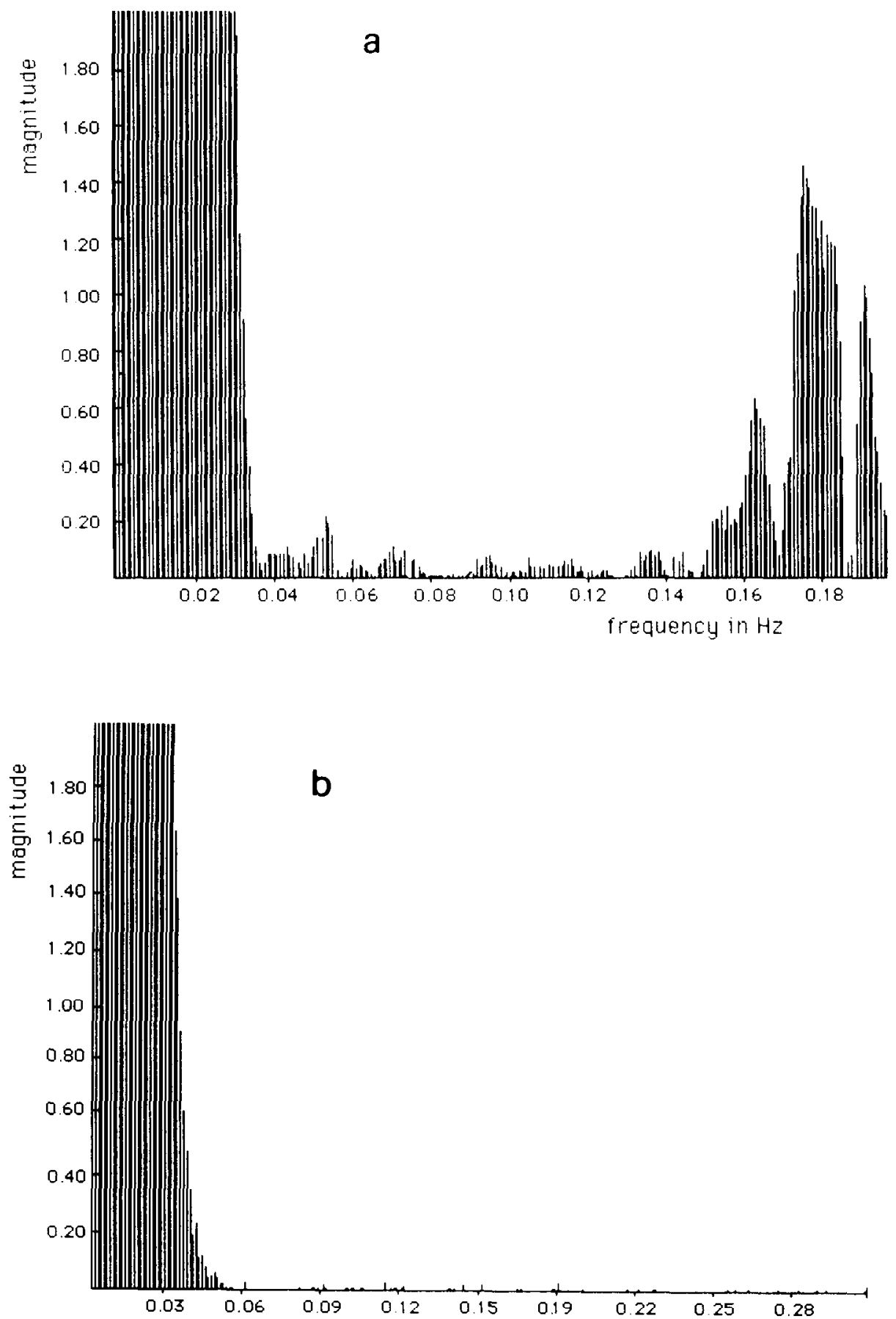

Fig. 2. 


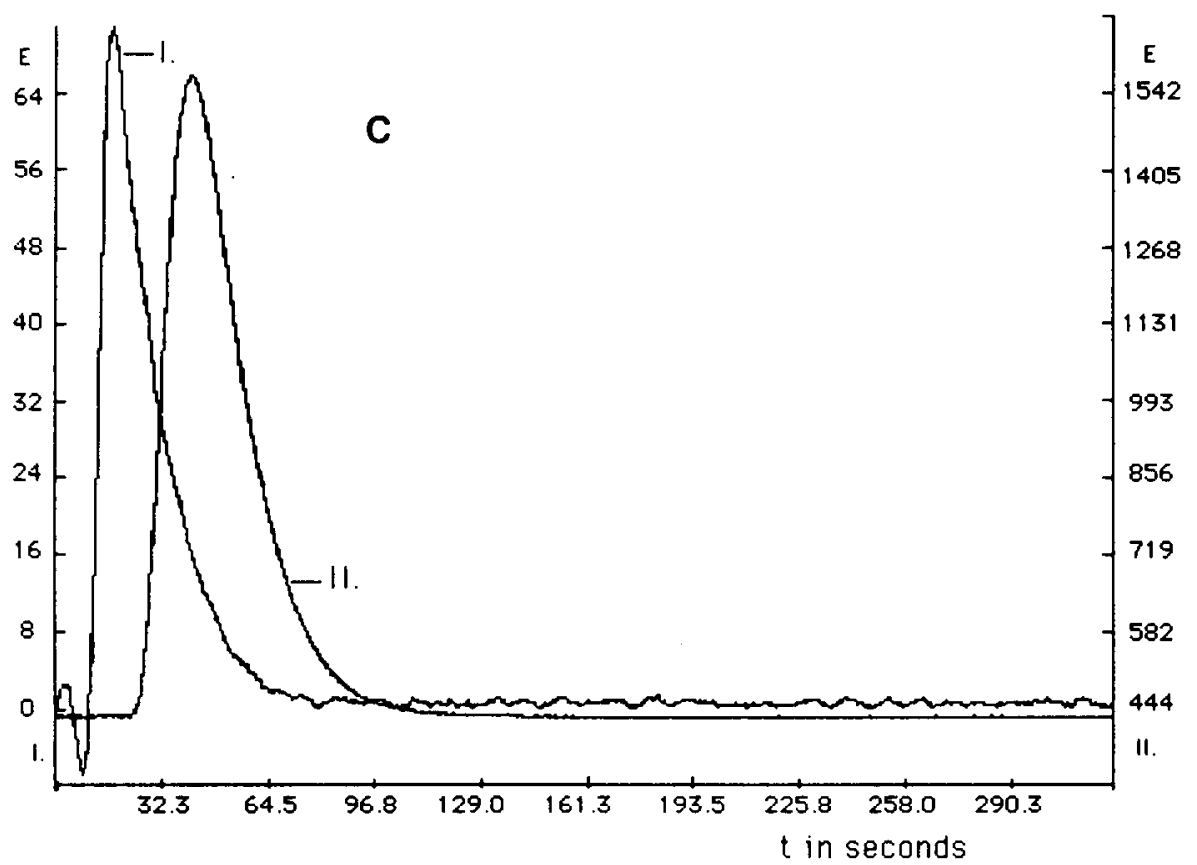

Fig. 2 (continued). Magnitude spectra of the signals from the Skalar spectrophotometer, the signal being measured (a) every $1.00 \mathrm{~s}$ and (b) every $0.63 \mathrm{~s}$. (c) Impulse-response function of the Skalar spectrophotometer (1), the detector signals being measured about every $0.63 \mathrm{~s}$; the function is compared with the direct response of the Skalar spectrophotometer (II). Flow-rate, $0.79 \mathrm{ml} \mathrm{min}^{-1}$; concentration of sample, $0.6 \times 10^{-4} \mathrm{M}$.

the fast Fourier transformation 512 points were used.

\section{RESULTS AND DISCUSSION}

\section{Spectrophotometer (Skalar)}

The signals from the spectrophotometer and the conductivity cell were measured every $1.00 \mathrm{~s}$. The magnitude spectrum of the signals from the spectrophotometer is shown in Fig. 2a. The aliasing effect of the electronic chopper of the spectrophotometer causes the coloured noise. Aliasing occurs if the sample frequency is too low. This chopping is achieved by on- and off-switching of the lamp. According to the Skalar manual, this should have a cycle of $650 \mathrm{~ms}$. By synchronizing the signal measurement with the lamp current, a better result was obtained (Fig. 2b). The correct measurement frequency appeared to be $1.58 \mathrm{~s}^{-1}$.

The impulse-response function of the Skalar spectrophotometer is depicted in Fig. 2c together with the direct response of the manifold as measured by the photometer. It can be seen that the contribution of the detector in this manifold to the peak broadening is considerable. The peak broadening factor is expressed as the value determined by dividing the baseline-to-baseline time of the impulse-response function by the baseline-tobaseline time of the direct response function. In this instance the peak broadening factor is 0.6 . The peak broadening factor is a measure for the contribution of the detector to the width of the peak and depends on the manifold used. Therefore, the same manifold has to be used for the determination in order to compare the factors of different detectors. Note that the total peak is determined by the convolution of the different impulse-response functions and not by multiplication. From the exponential decrease in the impulse-response function it can be concluded that the photometer cell seems to act like a single well 
stirred tank, so the construction of the detector cell can be improved by simply reducing the volume. The smallest possible size of a detector cell is determined primarily by the molar absorptivity and the number of photons passing per second through the cell. The limit with a standard optimized spectrophotometer is about $10 \mu \mathrm{l}$ [5].

The shape and height of the impulse-response function do not depend on the concentration, which means that the detector response is linear in this concentration range. On increasing the flow, the impulse-response function became narrower and higher. However, the peak broadening factor did not change.

\section{Spectrophotometer (Zeiss) with Hellma U-shaped cell}

The signals from the detectors were measured every $0.36 \mathrm{~s}$. According to the magnitude spectrum of the transformed signal of the spectrophotometer, there is no coloured noise. The magnitude of the white noise is high. The impulse-response function and the direct response of the manifold as measured by the photometer are depicted in Fig. 3. It can be seen that the peak broadening caused by the Hellma cell is larger than that from the cell in the Skalar spectrophotometer, the peak broadening factor being 0.8 for an otherwise identical manifold. Also, the Hellma cell seems to act like a well stirred tank and the construction can therefore be optimized by reducing the volume.

The impulse-response function has a similar behavioural pattern to that with the Skalar spectrophotometer.

\section{Wall-jet system}

The wall-jet potentiometric system at constant current consists of a Metrohm cell [8] with a gold working electrode and a glassy carbon counter electrode. The constant current through these electrodes was $10 \mu \mathrm{A}$. The signals from the detectors were measured every $0.50 \mathrm{~s}$. According to the

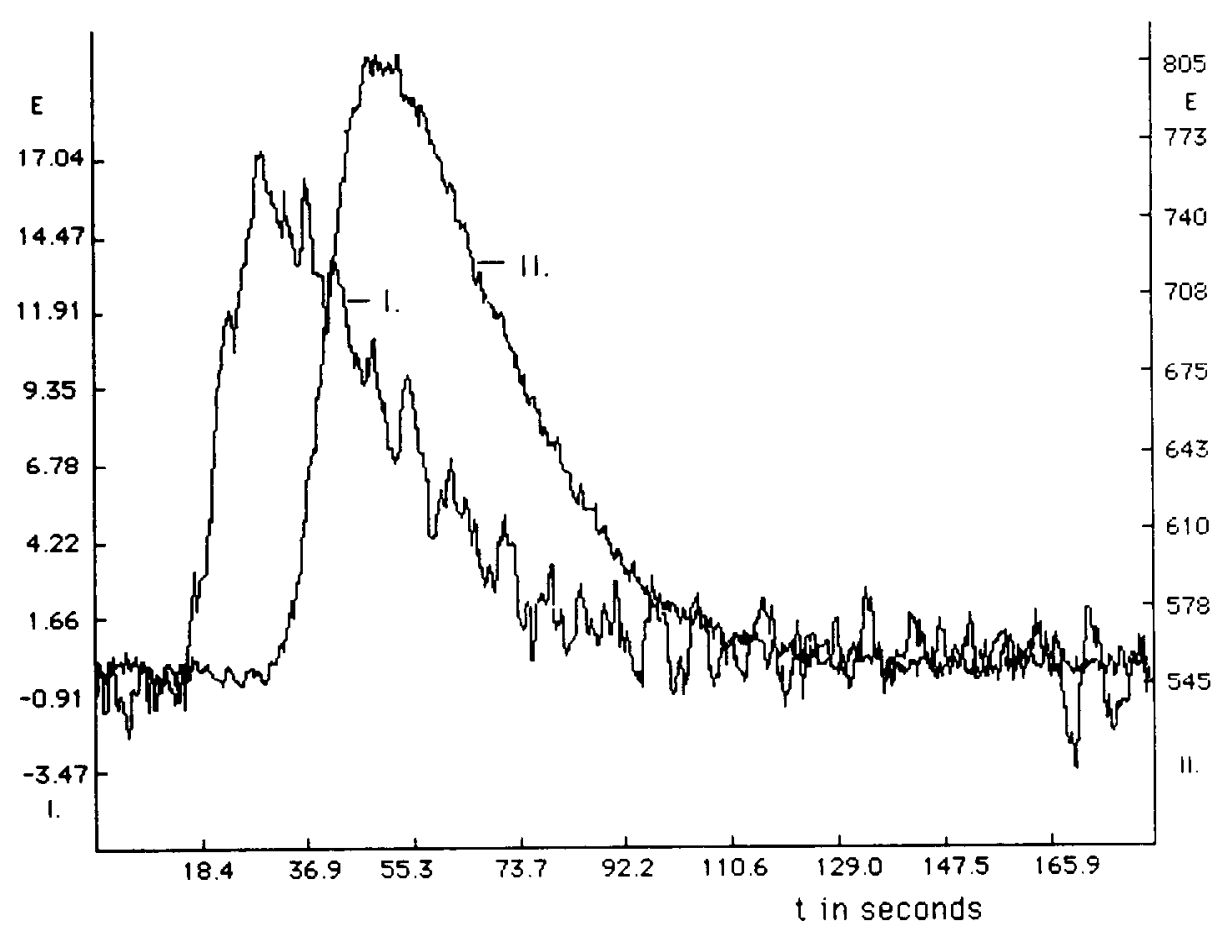

Fig. 3. Impulse-response function of the Zeiss spectrophotometer with Hellma U-shaped cell (I), the detector signals being measured every $0.36 \mathrm{~s}$; the function is compared with the direct response of the Zeiss spectrophotometer (II). Flow-rate, $1.05 \mathrm{ml} \mathrm{min}^{-1}$; concentration of sample, $0.5 \times 10^{-4} \mathrm{M}$. 
magnitude spectrum there was no coloured noise.

The impulse-response function and the direct response of the manifold as measured by the walljet system are depicted in Fig. 4. The peak broadening factor in this manifold is 0.5 . The impulseresponse function exists of two parts. The first is a sharp peak which is caused by the direct flow towards the electrode. The second part is caused by the washing out of the analyte of the detector cell, i.e., the analyte recirculates towards the electrode surface. For improvements in the construction, the detector cell must be changed in such a way that this recirculation of the analyte is prevented; an example of such a construction is the confined wall jet, described by Dalhuysen et al. [9].

The shape of the impulse-response function of this detector was also independent of the concentration and became higher and narrower with increasing flow. The peak broadening factor did not change.

\section{pH electrode system}

A glass electrode and a calomel reference electrode are used in the Metrohm cell [8]. The signals from the detectors were measured every $0.60 \mathrm{~s}$. No coloured noise was found in the magnitude spectrum. An impulse-response function and the direct response of the manifold as measured by the $\mathrm{pH}$ electrode system are depicted in Fig. 5. The peak broadening factor was 0.6 . The response of the glass electrode is logarithmically dependent on the concentration. The shape of the impulse-response function changes with the concentration in such a way that the former becomes higher on lowering the concentration.

The logarithmic effect probably divides the impulse-response function into two peaks and provides one or more small peaks in the last part. This effect is further considered in the Appendix. Despite the influences of the logarithmic effect, one can conclude from the flow tests that the impulse-response function exhibits two features,

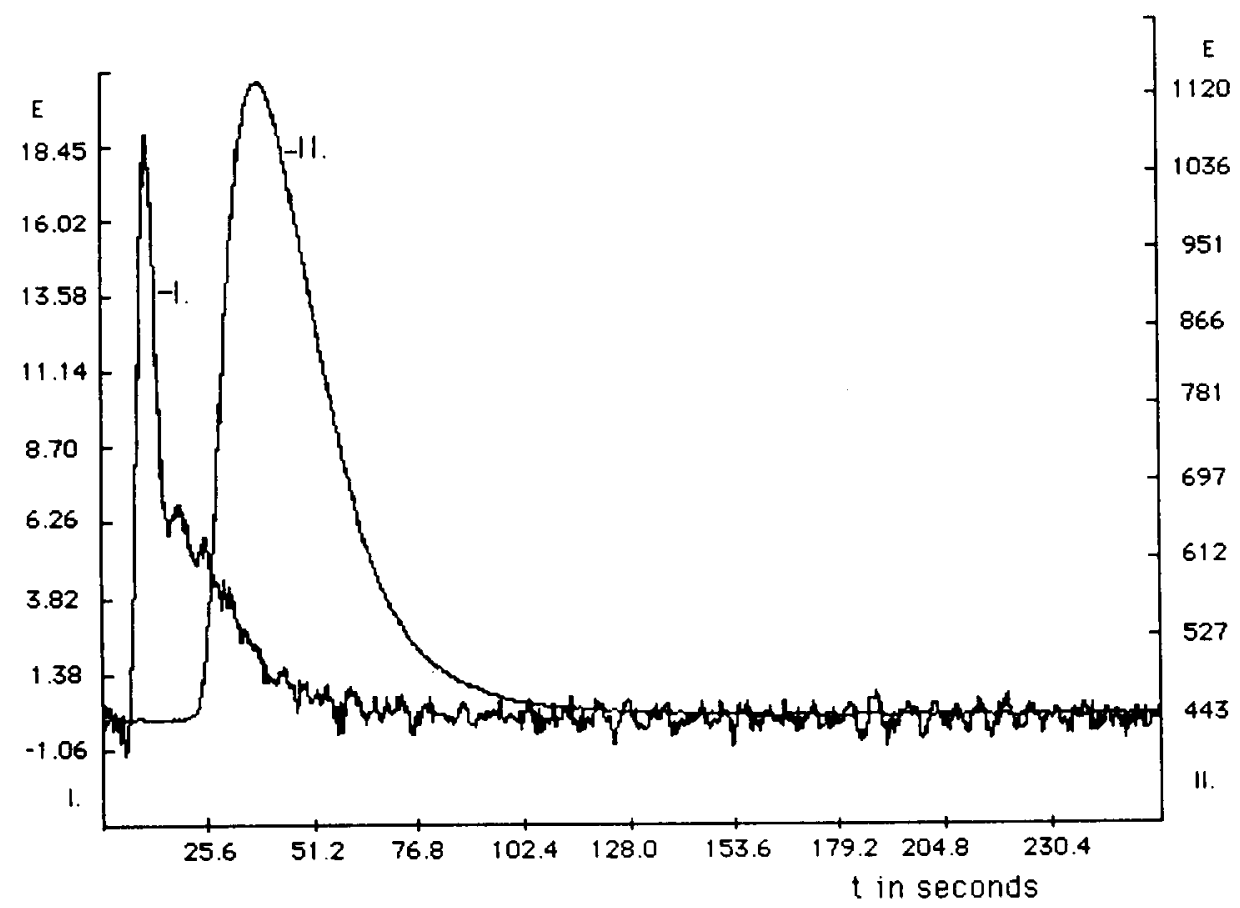

Fig. 4. Impulse-response function of the wall-jet system (I) with glassy carbon working electrode and gold reference electrode, the detector signals being measured every $0.50 \mathrm{~s}$; the function is compared with the direct response of the wall-jet system (II). Flow-rate, $1.00 \mathrm{ml} \mathrm{min}^{-1}$; concentration of sample, $2.5 \times 10^{-4} \mathrm{M}$. 


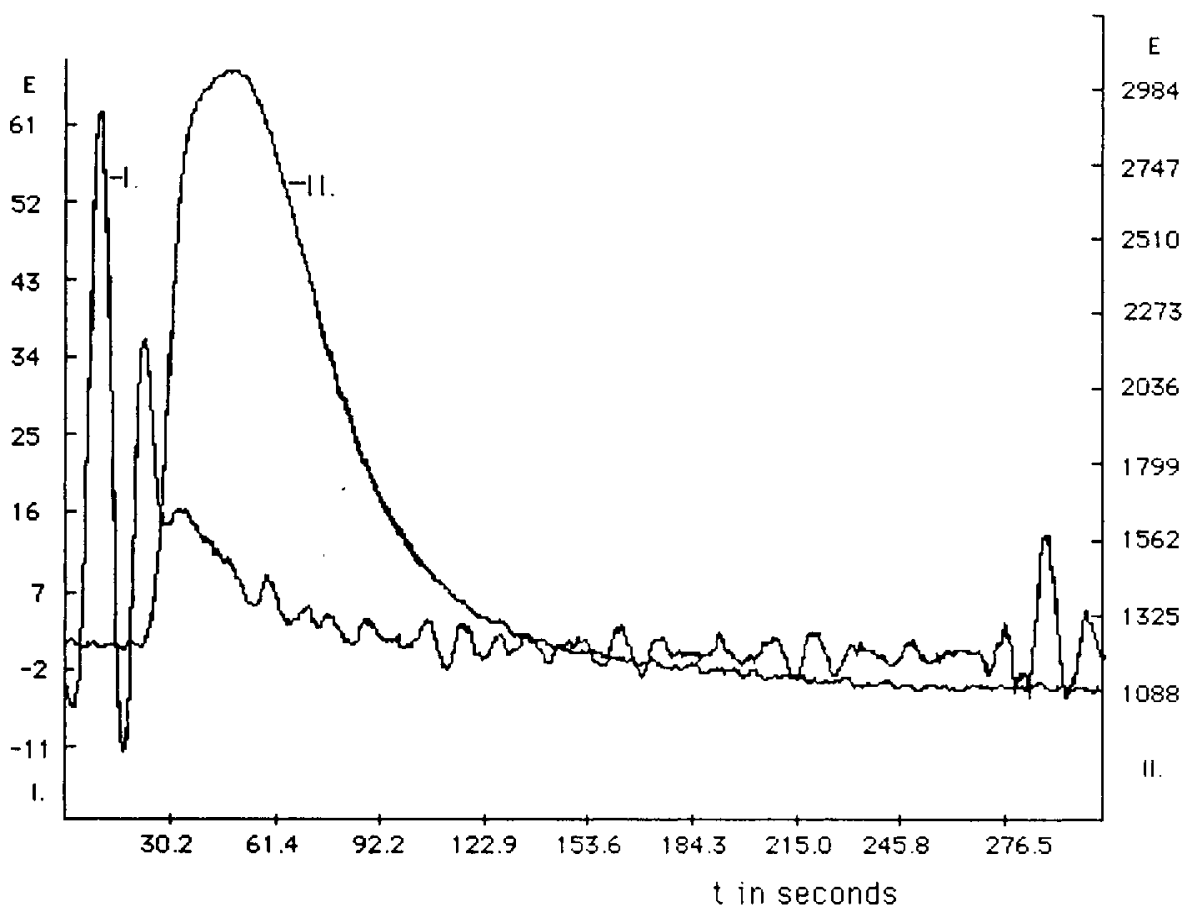

Fig. 5. Impulse-response function of the glass electrode system (I), the detector signals being measured every $0.60 \mathrm{~s}$; the function is compared with the direct response function. Flow-rate, $1.00 \mathrm{ml} \mathrm{min}-1$; concentration of sample, $5.0 \times 10^{-4} \mathrm{M}$.

namely direct flow towards the electrode and washing out of the analyte from the detector cell. Increasing the flow again caused a higher and narrower impulse-response function. The peak broadening factor did not change.

\section{Potassium ISFET}

The impulse-response function of a detector cell containing a potassium ion-sensitive field effect transistor (ISFET) and a reference ISFET was determined. The device is provided with a potassium-selective membrane, i.e., silicone rubber with valinomycin as the ionophore, which is chemically bonded to the silica of the transistor. The signals from the conductivity cell and the ISFET were measured every $0.2 \mathrm{~s}$.

The magnitude spectrum of the response of the ISFET showed coloured noise. On increasing the flow by changing the pump speed, this coloured noise shifted in the magnitude spectrum. On increasing the flow by changing the diameters of the pump tubes, the frequency of the coloured noise did not change. Hence this noise was caused by the pump. By using pulse dampers the magnitude of this coloured noise was halved.

The impulse-response function and the direct response of the manifold as measured by the ISFET system are depicted in Fig. 6. The peak broadening factor is 0.7 . The response of the potassium ISFET is logarithmically dependent on the concentration. The function becomes higher with lower concentration and the logarithmic effect, i.e., the division of the function into two peaks, becomes less. The detector seems to act more linearly with lower concentrations, as shown in Fig. 6. On increasing the flow, the general shape of the peak did not change but became higher and narrower. The peak broadening factor did not change.

\section{Sodium ISFET}

The impulse-response function of a detector cell containing a sodium ISFET was determined. There is no membrane bonded to the silica of the transistor. The signals from the conductivity cell and the ISFET were measured every $0.2 \mathrm{~s}$. The 


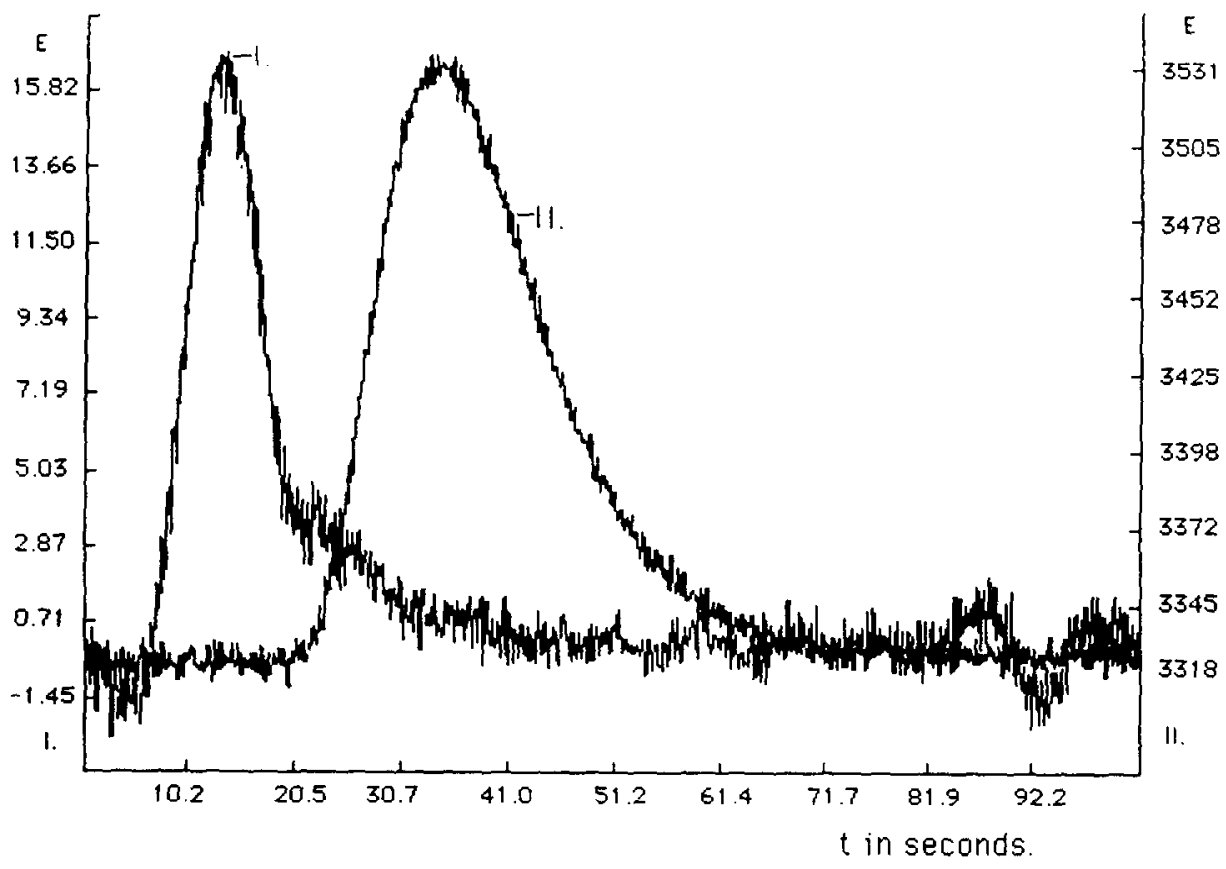

Fig. 6. Impulse-response function of the ISFET system (I) with the potassium ISFET, the detector signals being measured every 0.20 $\mathrm{s}$; the function is compared with the direct response of the potassium ISFET system (II). Flow-rate, $0.92 \mathrm{ml} \mathrm{min}^{-1}$; concentration of sample, $2.5 \times 10^{-3} \mathrm{M}$.

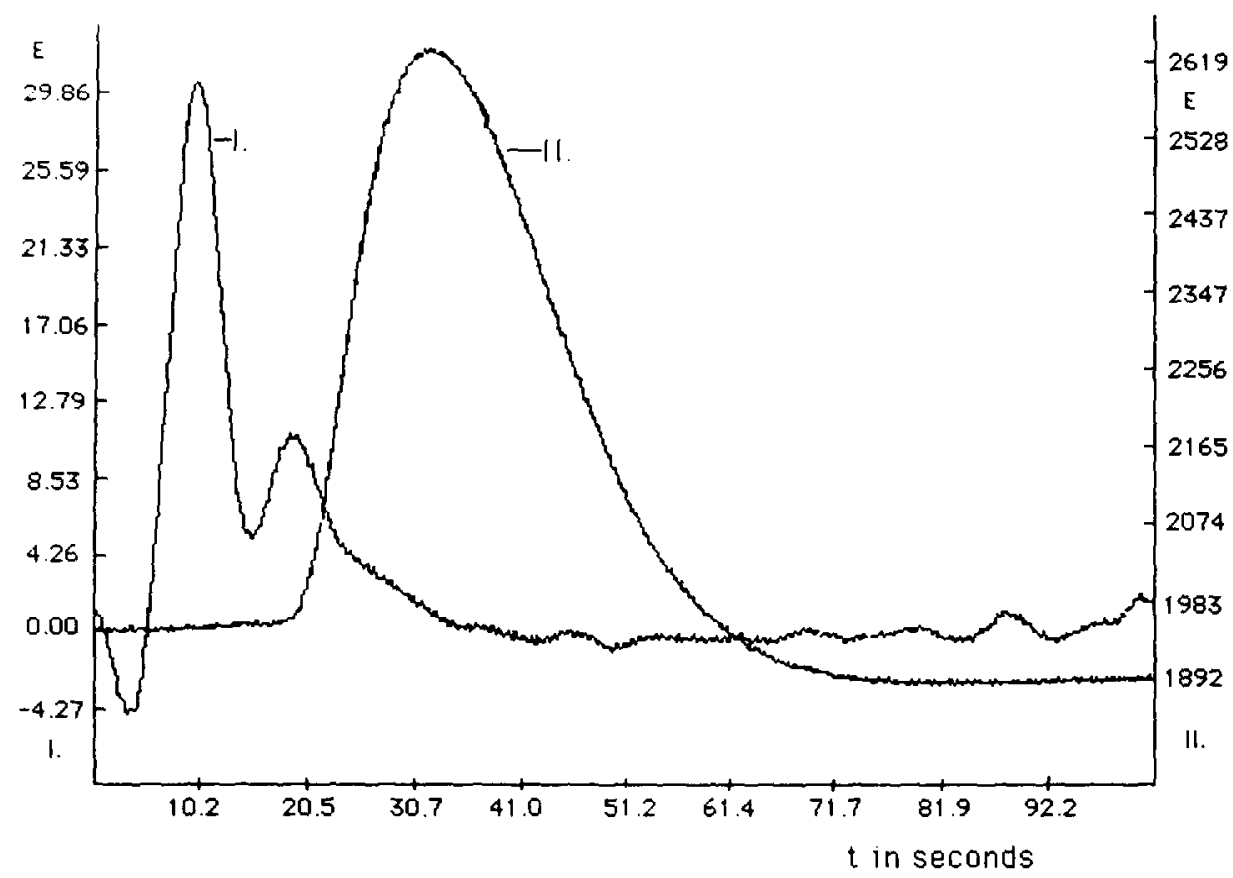

Fig. 7. Impulse-response function of the ISFET system (I) with the sodium ISFET, the detector signals being measured every 0.20 s; the function is compared with the direct response of the sodium ISFET system (II). Flow-rate, $0.92 \mathrm{ml}^{\text {min }}{ }^{-1}$; concentration of sample, $0.01 \mathrm{M}$. 
magnitude spectrum showed coloured noise caused by the pump with a magnitude of one. The magnitude of the white noise was lower than that with the potassium ISFET.

The impulse-response function and the direct response function of the manifold as measured by the ISFET system are shown in Fig. 7. The peak broadening factor was 0.7 . The response of the ISFET is logarithmically dependent on the concentration. The impulse-response function in this instance is calculated with higher concentrations, so the two peaks caused by the logarithmic effect are more distinct. On increasing the flow, the general shape of the impulse-response function did not change but it became higher and narrower. The peak broadening factor did not change.

Despite the logarithmic effect, it can be seen that the ISFET is influenced by the washing out of the analyte from the detector cell. When the construction of the cell is optimized, this feature must be considered.

The potassium ISFET is more affected by coloured and white noise than the sodium ISFET. This may be caused by the membrane. The re- sponse of the sodium ISFET is faster. The time between $t=0$ and the peak maximum of the impulse-response function can be taken as the response time. When the response times of two detectors are compared in this way, it is important to keep the distance between the detector and the conductivity cell the same and to determine the response functions of the detectors at the same flow.

\section{Conclusions}

The impulse-response function of an ideal detector is high and narrow. A spectrophotometer accomplishes this when the volume of the flowthrough cell is small. With the wall-jet systems and the ISFETs it is important that the signal is only generated by the flow against the electrode and is not influenced by recirculation of the analyte in the detector cell.

The authors thank P.D. van der Wal for placing the ISFETs at their disposal. These investigations were supported (in part) by the Netherlands Foundation for Chemical Research (SON) with

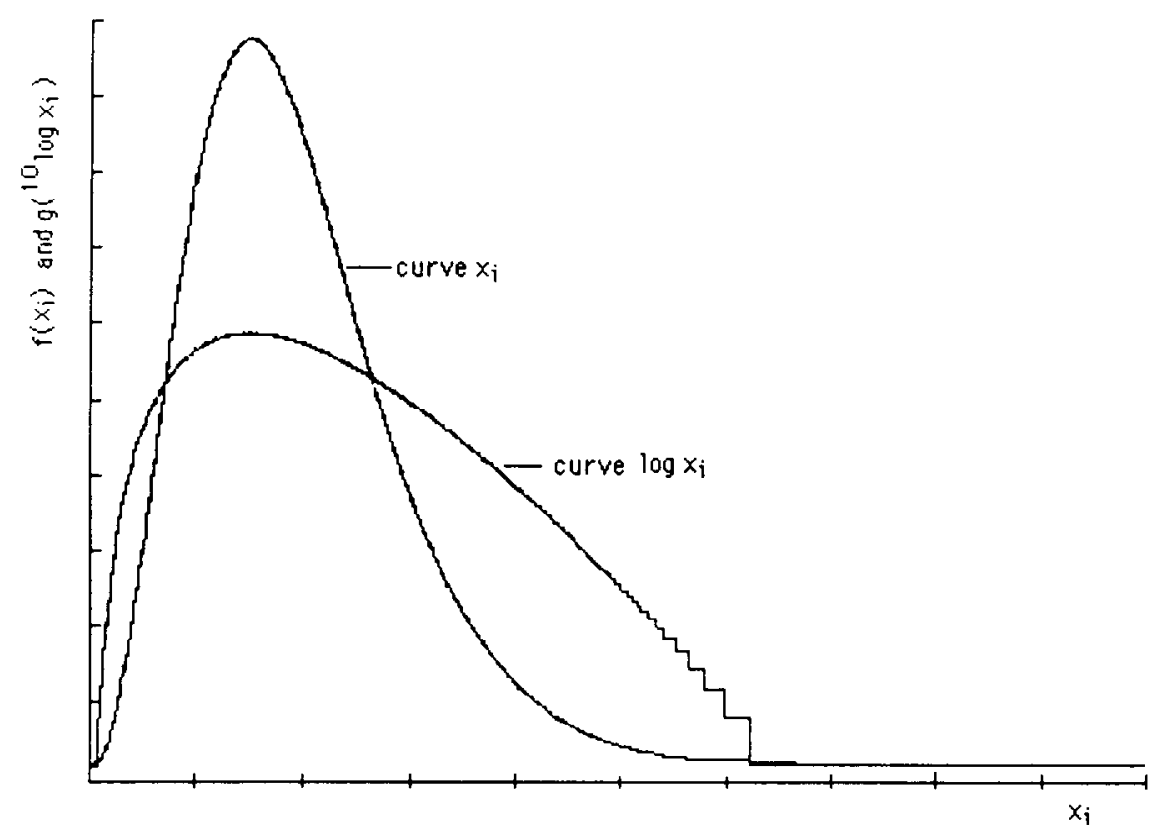

Fig. A1. The calculated curve and its logarithmic form. 


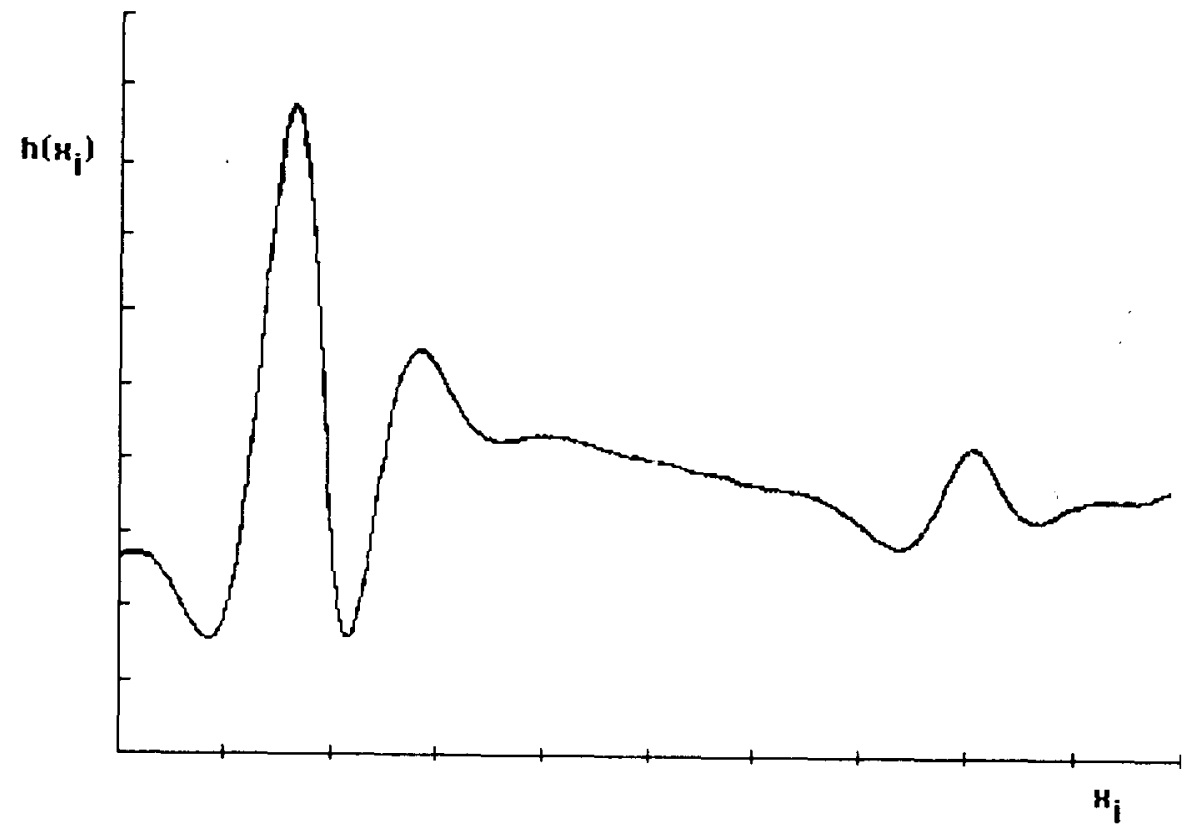

Fig. A2. Deconvolution and inverse FFT of the logarithmic calculated curve and the calculated curve.

financial aid from the Netherlands Technology Foundation.

\section{APPENDIX}

The ideal detector for flow systems should have a linear response over a wide concentration range, but detectors such as $\mathrm{pH}$ electrodes and ISFETs, whose responses are logarithmically dependent on the concentration, are also used as detectors for flow systems. To determine the effect of this logarithmic dependence on the impulse-response function, a curve $x_{i}$ and the logarithmically transformed curve $\log x_{i}$ are calculated. After scaling the curves so that the areas under the curves become uniform (Fig. A1), the logarithmic curve is deconvoluted by the original curve. The result is depicted in Fig. A2. The first peaks cause the splitting of the peak of the impulse-response function of the detectors with a logarithmic response. Also the last peaks of the impulse-response function of these detectors are caused by the logarithmic effect.

\section{REFERENCES}

1 I.C. van Nugteren-Osinga, M. Bos and W.E. van der Linden, Anal. Chim. Acta, 214 (1988) 77.

2 I.C. van Nugteren-Osinga, M. Bos and W.E. van der Linden, Anal. Chim. Acta, 222 (1989) 151.

3 D. Taylor and T.A. Nieman, Anal. Chim. Acta, 159 (1984) 397.

4 J. Ruzicka and E.H. Hansen, Anal. Chim. Acta, 99 (1978) 37.

5 H. Poppe, Anal. Chim. Acta, 145 (1983) 17.

6 P.D. van der Wal, H. Skowronska-Ptasinka, A. van der Berg, P. Bergveld, E.J.R. Sudhölter and D.N. Reinhoudt. Anal. Chim. Acta, 230 (1990) 59.

7 P.D. van der Wal, E.J.R. Sudhölter and D.N. Reinhoudt, in preparation.

8 M. Valcárcel and M.D. Luque de Castro, Flow Injection Analysis, Principles and Applications, Horwood, Chichester, 1987.

9 A.J. Dalhuysen, Th.H. van der Meer, C.J. Hoogendoorn, J.C. Hoogvliet and W.P. van Bennekom, J. Electroanal. Chem., 182 (1985) 295. 